Mathematical Sciences And Applications E-Notes

Volume 3 No. 1 PP. 126-136 (2015) @ MSAEN

\title{
ON THE RATE OF CONVERGENCE OF THE STANCU TYPE BERNSTEIN OPERATORS FOR FUNCTIONS OF BOUNDED VARIATION
}

\author{
RÜYA ÜSTER AND ERTAN IBBIKLI \\ (Communicated by Xiao-Jun YANG)
}

\begin{abstract}
In this paper, we estimate the rate of pointwise convergence of the Stancu type Bernstein operators for functions defined on the interval. To prove our main result, we have used some methods and techniques from probability theory.
\end{abstract}

\section{INTRODUCTION}

Let $B V(I)$ denote the class of functions that are of bounded variation on a set $I \subset \mathbb{R}$. Recently, some authors studied some linear positive operators and obtained the rate of convergence for functions in $B V(I)$. For example, Bojanic and Vuilleumier [1] estimated the rate of convergence of Fourier-Legendre series for functions of bounded variation on the interval $[0,1]$, Cheng [2] estimated the rate of convergence of Bernstein polynomials for functions bounded variation on $[0,1]$, and Zeng and Chen [3] and Guo [4] estimated the rate of convergence of Durrmeyer type operators for functions of bounded variation again on $[0,1]$.

For a function defined on the interval $[0,1]$, Bernstein operators $B_{n}(f), n \geq 1$, are defined by

$$
B_{n}(f ; x)=\sum_{k=0}^{n} f\left(\frac{k}{n}\right) p_{n k}(x), n \geq 1
$$

Date: Received: November 15, 2014; Accepted: December 20, 2014.

2010 Mathematics Subject Classification. 41A25; 41A35; 41A36.

Key words and phrases. Approximation; Bernstein-Stancu polynomials; Bounded variation; Total variation; Rate of convergence.

This article is the written version of author's plenary talk delivered on August 25-28, 2014 at 3rd International Eurasian Conference on Mathematical Sciences and Applications IECMSA-2014 at Vienna, Austria. 
where $p_{n k}(x)=\left(\begin{array}{l}n \\ k\end{array}\right) x^{k}(1-x)^{n-k}$ is the Bernstein basis $(0 \leq x \leq 1)$.

The operators defined by (1) were introduced by Bernstein [5] and studied by many authors.

The Stancu polynomials studied in this paper are given [6] by

$$
B_{n}^{(\alpha, \beta)}(f, x)=\sum_{k=0}^{n} p_{n k}(x) f\left(\frac{k+\alpha}{n+\beta}\right)
$$

where $\alpha, \beta$ are real parameters $(0 \leq \alpha \leq \beta)$ and $x \in[0,1]$.

In this paper, by means of techniques of probability theory and methods of Bojanic and Vuilleumier [1], Cheng [2], Zeng and Chen [3] we shall estimate the rate of convergence of the operators $B_{n}^{(\alpha, \beta)}(f, x)$ for functions of bounded variation.

Theorem 1.1. Let $f$ be a function of bounded variation on $[0,1]$. Then for every $x \in(0,1)$, we have

$$
\begin{aligned}
\left|B_{n}^{(\alpha, \beta)}(f, x)-\frac{1}{2}(f(x+)+f(x-))\right| \leq & 3 \frac{(\alpha-\beta x)^{2}+n x(1-x)}{(n+\beta)^{2} x^{2}(1-x)^{2}} \\
& \times\left\{\sum_{k=1}^{n} \bigvee_{x-x / \sqrt{k}}^{x+(1-x) / \sqrt{k}}\left(g_{x}\right)\right\} \\
& +\frac{1}{\sqrt{n x(1-x)}}[|f(x+)-f(x-)| \\
& \left.+|f(x)-f(x-)| e_{n}(x) \frac{1}{\sqrt{2 e}}\right]
\end{aligned}
$$

where $e_{n}(x)=\left\{\begin{array}{cc}1, & x=k^{\prime} \text { for some } k^{\prime} \in \mathbb{N} \\ 0, & x \neq k^{\prime} \text { for all } k^{\prime} \in \mathbb{N}\end{array}, e=2.71 \ldots\right.$ and $\bigvee_{a}^{b}\left(g_{x}\right)$ is the total variation of $g_{x}$ on $[a, b]$,

$$
g_{x}(t)=\left\{\begin{array}{cc}
f(t)-f(x+) & x<t \leq 1 \\
0 & t=x \\
f(t)-f(x-) & 0 \leq t<x
\end{array} .\right.
$$

\section{AUXILIARY RESULT}

In this section, we give certain results that are neccessary to prove our main theorems.

Lemma 2.1. For $B_{n}^{(\alpha, \beta)}\left(t^{s} ; x\right), s=0,1,2$, one has

$$
\begin{gathered}
B_{n}^{(\alpha, \beta)}(1, x)=1 \\
B_{n}^{(\alpha, \beta)}(t, x)=x+\frac{\alpha-\beta x}{n+\beta} \\
B_{n}^{(\alpha, \beta)}\left(t^{2}, x\right)=x^{2}+\frac{(\alpha-\beta x)(2 n x+\beta x+\alpha)+n x(1-x)}{(n+\beta)^{2}} .
\end{gathered}
$$

Proof.

$$
B_{n}^{(\alpha, \beta)}(1, x)=\sum_{k=0}^{n} p_{n k}(x)=B_{n}(1, x)=1
$$




$$
\begin{aligned}
B_{n}^{(\alpha, \beta)}(t, x)= & \sum_{k=0}^{n} \frac{k+\alpha}{n+\beta} p_{n k}(x) \\
= & \frac{n}{n+\beta} \sum_{k=0}^{n} \frac{k}{n} p_{n k}(x)+\frac{\alpha}{n+\beta} \sum_{k=0}^{n} p_{n k}(x) \\
= & \frac{n}{n+\beta} B_{n}(t, x)+\frac{\alpha}{n+\beta} B_{n}(1, x) \\
= & \frac{n x}{n+\beta}+\frac{\alpha}{n+\beta}=x+\frac{\alpha-\beta x}{n+\beta} \\
B_{n}^{(\alpha, \beta)}\left(t^{2}, x\right)= & \sum_{k=0}^{n}\left(\frac{k+\alpha}{n+\beta}\right)^{2} p_{n k}(x) \\
= & \frac{n^{2}}{(n+\beta)^{2}} \sum_{k=0}^{n}\left(\frac{k}{n}\right)^{2} p_{n k}(x) \\
& +\frac{2 \alpha n}{(n+\beta)^{2}} \sum_{k=0}^{n} \frac{k}{n} p_{n k}(x)+\frac{\alpha^{2}}{(n+\beta)^{2}} \sum_{k=0}^{n} p_{n k}(x) \\
= & \frac{n^{2}}{(n+\beta)^{2}} B_{n}\left(t^{2}, x\right)+\frac{2 \alpha n}{(n+\beta)^{2}} B_{n}(t, x) \\
& +\frac{\alpha^{2}}{(n+\beta)^{2}} B_{n}(1, x) \\
= & x^{2}+\frac{(\alpha-\beta x)(2 n x+\beta x+\alpha)+n x(1-x)}{(n+\beta)^{2}} .
\end{aligned}
$$

By direct calculation, we also find the following equalities;

$$
B_{n}^{(\alpha, \beta)}\left((t-x)^{2}, x\right)=\frac{(\alpha-\beta x)^{2}+n x(1-x)}{(n+\beta)^{2}} .
$$

Lemma 2.2. For all $x \in(0,1)$, we have

(5) $\quad \lambda_{n}(x, t):=\int_{0}^{t} K_{n}(x, u) d u \leq \frac{1}{(x-t)^{2}} \frac{(\alpha-\beta x)^{2}+n x(1-x)}{(n+\beta)^{2}}, 0<t<x$

and

(6) $1-\lambda_{n}(x, z):=\int_{z}^{1} K_{n}(x, u) d u \leq \frac{1}{(z-x)^{2}} \frac{(\alpha-\beta x)^{2}+n x(1-x)}{(n+\beta)^{2}}, x \leq z<1$

where

$$
K_{n}(x, t)=\left\{\begin{array}{cc}
\sum_{k+\alpha \leq(n+\beta) t} P_{n k}(x) & 0<t \leq 1 \\
0 & t=0
\end{array} .\right.
$$


Proof. First we prove (5)

$$
\begin{aligned}
\lambda_{n}(x, t) & =\int_{0}^{t} K_{n}(x, u) d u \\
& \leq \int_{0}^{t} K_{n}(x, u)\left(\frac{x-u}{x-t}\right)^{2} d u \\
& =\frac{1}{(x-t)^{2}} B_{n}^{(\alpha, \beta)}\left((u-x)^{2}, x\right) .
\end{aligned}
$$

By (4), we get

$$
\lambda_{n}(x, t) \leq \frac{1}{(x-t)^{2}} \frac{(\alpha-\beta x)^{2}+n x(1-x)}{(n+\beta)^{2}} .
$$

The proof of (6) is similar.

Lemma 2.3. Let $\xi_{1}$ be a sequence of independent random variables with two point binomial distribution $P\left(\xi_{1}=k\right):=x^{k}(1-x)^{1-k}(k=0,1$, and $0 \leq x \leq 1$ being a parameter). Then

$$
a_{1}=E \xi_{1}=x, E\left(\xi_{1}-a_{1}\right)^{2}=x(1-x)
$$

and

$$
E\left(\xi_{1}-a_{1}\right)^{3}=x(1-x)\left(2 x^{2}-2 x+1\right) .
$$

Proof. Let $\left\{\xi_{1}\right\}_{k=1}^{\infty}$ be a sequence of independent random variables identically distributed with $\xi_{1}, \eta_{n}=\sum_{k=1}^{n} \xi_{k}$. Then the probability distributions of the random variable $\eta_{n}$ is

$$
P\left(\mu_{n}=k\right)=p_{n k}(x)=\left(\begin{array}{l}
n \\
k
\end{array}\right) x^{k}(1-x)^{1-k},(0 \leq k \leq n) .
$$

For $M_{i}(x)=\sum_{k=0}^{1} k^{i} x^{k}(1-x)^{1-k}$, we find that

$$
M_{0}(x)=1, M_{1}(x)=x, M_{2}(x)=x, M_{3}(x)=x .
$$

From the definition of expectation, we get $E\left(\xi_{1}\right)=M_{1}(x)=x$. Also

$$
\begin{gathered}
E\left(\xi_{1}-a_{1}\right)^{2}=\sum_{j=0}^{2}\left(\begin{array}{l}
2 \\
j
\end{array}\right)(-1)^{j} M_{2-j}\left[M_{1}(x)\right]^{j}=x(1-x) \\
E\left(\xi_{1}-a_{1}\right)^{3}=\sum_{j=0}^{3}\left(\begin{array}{l}
3 \\
j
\end{array}\right)(-1)^{j} M_{3-j}\left[M_{1}(x)\right]^{j}=x(1-x)\left(2 x^{2}-2 x+1\right) .
\end{gathered}
$$

Lemma 2.4. (Berry-Esseen). Let $\left\{\xi_{1}\right\}_{k=1}^{\infty}$ be a sequence of independent and identically distributed random variable with finite variance such that the expectation 
$E\left(\xi_{1}\right):=a_{1} \in \mathbb{R}$, the variance $\operatorname{Var}\left(\xi_{1}\right):=E\left(\xi_{1}-a_{1}\right)^{2}=b_{1}^{2}>0$ and $E\left|\xi_{1}-E\left(\xi_{1}\right)\right|^{3}<$ $\infty$. Then there exist a constant $C, \frac{1}{\sqrt{2 \pi}} \leq C<0.82$, such that for all $n$ and $t$

$$
\left|P\left(\frac{1}{b_{1} \sqrt{n}} \sum_{k=1}^{n}\left(\xi_{1}-a_{1}\right) \leq t\right)-\frac{1}{\sqrt{2 \pi}} \int_{-\infty}^{t} e^{-u^{2} / 2} d u\right|<C \frac{E\left|\xi_{1}-E\left(\xi_{1}\right)\right|^{3}}{b_{1}^{3} \sqrt{n}} .
$$

Its proof can be found in Shiryayev [7].

Lemma 2.5. For all $x \in[0,1]$, we have

$$
\left|\left(\sum_{(n+\beta) x<k+\alpha \leq n} p_{n k}(x)\right)-\frac{1}{2}\right| \leq \frac{0,8\left(2 x^{2}-2 x+1\right)}{\sqrt{n x(1-x)}} \leq \frac{1}{\sqrt{n x(1-x)}} .
$$

Proof. From Lemma 2.3 for $P\left(\mu_{n}=k\right)=p_{n k}(x)=\left(\begin{array}{l}n \\ k\end{array}\right) x^{k}(1-x)^{1-k},(0 \leq k \leq n)$

$$
\begin{aligned}
\sum_{(n+\beta) x<k+\alpha \leq n} p_{n k}(x) & =P\left((n+\beta) x<\mu_{n} \leq n\right) \\
& =1-P\left(\mu_{n} \leq(n+\beta) x\right) \\
& =1-P\left(\frac{\mu_{n}-(n+\beta) x}{\sqrt{n x(1-x)}} \leq 0\right)
\end{aligned}
$$

then

$$
\begin{aligned}
\left|\left(\sum_{(n+\beta) x<k+\alpha \leq n} P_{n k}(x)\right)-\frac{1}{2}\right| & =\left|P\left(\frac{\mu_{n}-(n+\beta) x}{\sqrt{n x(1-x)}} \leq 0\right)-\frac{1}{2}\right| \\
& <C \frac{E\left|\xi_{1}-E\left(\xi_{1}\right)\right|^{3}}{b_{1}^{3} \sqrt{n}} \\
& <\frac{0.8\left(2 x^{2}-2 x+1\right)}{\sqrt{n x(1-x)}} \leq \frac{1}{\sqrt{n x(1-x)}} .
\end{aligned}
$$

Lemma 2.6. For all $x \in[0,1]$, we have

$$
B_{n}^{(\alpha, \beta)}(\operatorname{sgn}(t-x), x)=2 \sum_{(n+\beta) x<k+\alpha \leq n} P_{n k}(x)-1+e_{n}(x) P_{n k}(x) .
$$

Proof. One has

$$
\begin{aligned}
B_{n}^{(\alpha, \beta)}(\operatorname{sgn}(t-x), x) & =\sum_{k=0}^{n} \operatorname{sgn}\left(\frac{k+\alpha}{n+\beta}-x\right) p_{n k}(x) \\
& =\sum_{(n+\beta) x<k+\alpha \leq n} p_{n k}(x)-\sum_{0<k+\alpha \leq(n+\beta) x} p_{n k}(x)
\end{aligned}
$$

and from (3), we can write

$$
\begin{aligned}
1= & B_{n}^{(\alpha, \beta)}(1, x)=\sum_{(n+\beta) x<k+\alpha \leq n} p_{n k}(x)+\sum_{0<k+\alpha \leq(n+\beta) x} p_{n k}(x) \\
& +e_{n}(x) p_{n k}(x) .
\end{aligned}
$$


Thus there follows

$$
\begin{aligned}
B_{n}^{(\alpha, \beta)}(\operatorname{sgn}(t-x), x)= & \sum_{(n+\beta) x<k+\alpha \leq n} p_{n k}(x) \\
& -\left[1-\sum_{(n+\beta) x<k+\alpha \leq n} p_{k}(x)-e_{n}(x) p_{n k}(x)\right] \\
= & 2 \sum_{(n+\beta) x<k+\alpha \leq n} P_{n k}(x)-1+e_{n}(x) P_{n k}(x) .
\end{aligned}
$$

Lemma 2.7. There holds the inequality

$$
\begin{aligned}
& \mid \frac{f(x+)-f(x-)}{2} B_{n}^{(\alpha, \beta)}(\operatorname{sgn}(t-x), x) \\
& +\left[f(x)-\frac{1}{2} f(x+)-\frac{1}{2} f(x-)\right] B_{n}^{(\alpha, \beta)}\left(\delta_{x}, x\right) \mid \\
\leq & \frac{1}{\sqrt{n x(1-x)}}\left[|f(x+)-f(x-)|+|f(x)-f(x-)| e_{n}(x) \frac{1}{\sqrt{2 e}}\right] .
\end{aligned}
$$

Proof. We have

$$
\begin{aligned}
& \mid \frac{f(x+)-f(x-)}{2} B_{n}^{\alpha, \beta}(\operatorname{sgn}(t-x), x) \\
& +\left[f(x)-\frac{1}{2} f(x+)-\frac{1}{2} f(x-)\right] B_{n}^{\alpha, \beta}\left(\delta_{x}, x\right) \mid \\
= & \mid \frac{f(x+)-f(x-)}{2}\left[2 \sum_{(n+\beta) x<k+\alpha \leq n} P_{n k}(x)-1+e_{n}(x) P_{n k}(x)\right] \\
\leq & \mid \frac{f(x+)-f(x-)}{2}\left[2 \sum_{(n+\beta) x<k+\alpha \leq n} P_{n k}(x)-1\right] \\
& +\left|[f(x)-f(x-)] e_{n}(x) p_{n k}(x)\right| .
\end{aligned}
$$

\section{PROOF OF THE THEOREM}

Now we can establish the theorem.

For any $f(t) \in B V[0,1]$, we decompose $f(t)$ into four parts as

$$
\begin{aligned}
f(t)= & \frac{f(x+)+f(x-)}{2}+g_{x}(t) \\
& +\frac{f(x+)-f(x-)}{2} \operatorname{sgn}(t-x) \\
& +\delta_{x}(t)\left(f(x)-\frac{f(x+)+f(x-)}{2}\right)
\end{aligned}
$$


where

$$
\delta_{x}(t)=\left\{\begin{array}{ll}
1, & x=t \\
0, & x \neq t
\end{array} .\right.
$$

If we apply $B_{n}^{(\alpha, \beta)}$ to both sides of (3.1), we have

$$
\begin{aligned}
B_{n}^{(\alpha, \beta)}(f, x)= & \frac{1}{2}(f(x+)+f(x-)) B_{n}^{(\alpha, \beta)}(1, x) \\
& +B_{n}^{(\alpha, \beta)}\left(g_{x}, x\right)+\frac{f(x+)-f(x-)}{2} B_{n}^{(\alpha, \beta)}(\operatorname{sgn}(t-x), x) \\
& +\left[f(x)-\frac{1}{2}(f(x+)+f(x-))\right] B_{n}^{(\alpha, \beta)}\left(\delta_{x}, x\right) .
\end{aligned}
$$

If we take the absolute values of the inequality $(3.2)$ and note $B_{n}^{(\alpha, \beta)}(1, x)=1$ by (4), we have

$$
\begin{aligned}
& \left|B_{n}^{(\alpha, \beta)}(f, x)-\frac{1}{2}(f(x+)+f(x-)) B_{n}^{(\alpha, \beta)}(1, x)\right| \\
\leq & \left|B_{n}^{(\alpha, \beta)}\left(g_{x}, x\right)\right| \\
& +\mid \frac{f(x+)-f(x-)}{2} B_{n}^{(\alpha, \beta)}(\operatorname{sgn}(t-x), x) \\
& +\left(f(x)-\frac{1}{2}(f(x+)+f(x-))\right) B_{n}^{(\alpha, \beta)}\left(\delta_{x}, x\right) \mid .
\end{aligned}
$$

First we estimate $B_{n}^{(\alpha, \beta)}\left(g_{x}, x\right)$ as follows:

$$
\left|B_{n}^{(\alpha, \beta)}\left(g_{x}, x\right)\right|=\left|\int_{0}^{1} g_{x}(t) d_{t} K_{n}(x, t) d t\right|,
$$

with the kernel $K_{n}(x, t)$ of (7). To estimate the integral of (8), we decompose it into three parts, as follows

$$
\begin{aligned}
& \int_{0}^{1} g_{x}(t) d_{t} K_{n}(x, t) d t \\
= & \left|\int_{0}^{x-x / \sqrt{n}}+\int_{x-x / \sqrt{n}}^{x+(1-x) / \sqrt{n}}+\int_{x+(1-x) / \sqrt{n}}^{1}\right| g_{x}(t) d_{t} K_{n}(x, t) d t \mid \\
\leq & \left|\int_{0}^{x-x / \sqrt{n}} g_{x}(t) d_{t} K_{n}(x, t) d t\right| \\
& +\left|\int_{x-x / \sqrt{n}}^{x+(1-x) / \sqrt{n}} g_{x}(t) d_{t} K_{n}(x, t) d t\right|+\mid \int_{x+(1-x) / \sqrt{n}}^{1} g_{x}(t) d_{t} K_{n}(x, t) d t \\
= & \left|I_{1}(n, x)\right|+\left|I_{2}(n, x)\right|+\left|I_{3}(n, x)\right| .
\end{aligned}
$$


and

$$
\begin{aligned}
I_{1}(n, x) \mid & =\left|\int_{0}^{x-x / \sqrt{n}} g_{x}(t) d_{t}\left(\lambda_{n}(x, t)\right) d t\right|, \\
\left|I_{2}(n, x)\right| & =\left|\int_{x-x / \sqrt{n}}^{x+(1-x) / \sqrt{n}} g_{x}(t) d_{t}\left(\lambda_{n}(x, t)\right) d t\right|
\end{aligned}
$$

$$
\left|I_{3}(n, x)\right|=\left|\int_{x+(1-x) / \sqrt{n}}^{1} g_{x}(t) d_{t}\left(\lambda_{n}(x, t)\right) d t\right|,
$$

$\lambda_{n}(x, t)$ being defined in (5).First we estimate $I_{2}(n, x)$. For $t \in\left[x-x / \sqrt{n}, x+\left(b_{n}-x\right) / \sqrt{n}\right]$, we have, as $g_{x}(x)=0$

$$
\begin{aligned}
\left|I_{2}(n, x)\right| & =\left|\int_{x-x / \sqrt{n}}^{x+(1-x) / \sqrt{n}}\left(g_{x}(t)-g_{x}(x)\right) d_{t}\left(\lambda_{n}(x, t)\right) d t\right| \\
& \leq \bigvee_{x-x / \sqrt{n}}^{x+(1-x) / \sqrt{n}}\left(g_{x}\right) \leq \frac{1}{n-1} \sum_{k=2}^{n} \bigvee_{x-x / \sqrt{k}}^{x+(1-x) / \sqrt{k}}\left(g_{x}\right) .
\end{aligned}
$$

Next, we estimate $I_{1}(n, x)$. Using partial Lebesgue-Stieltjes integration, we obtain

$$
\begin{aligned}
I_{1}(n, x)= & \int_{0}^{x-x / \sqrt{n}} g_{x}(t) d_{t}\left(\lambda_{n}(x, t)\right) d t \\
= & g_{x}\left(x-\frac{x}{\sqrt{n}}\right) \lambda_{n}(x, x-x / \sqrt{n})-g_{x}(0) \lambda_{n}(x, 0) \\
& -\int_{0}^{x-x / \sqrt{n}} \lambda_{n}(x, t) d_{t}\left(g_{x}(t)\right) d t .
\end{aligned}
$$

Because $\left|g_{x}\left(x-\frac{x}{\sqrt{n}}\right)\right|=\left|g_{x}\left(x-\frac{x}{\sqrt{n}}\right)-g_{x}(x)\right| \leq \bigvee_{x-x / \sqrt{n}}^{x}\left(g_{x}\right)$, it follows that

$$
\begin{aligned}
\left|I_{1}(n, x)\right| \leq & \bigvee_{x-x / \sqrt{n}}^{x}\left(g_{x}\right)\left|\lambda_{n}(x, x-x / \sqrt{n})\right| \\
& +\int_{0}^{x-x / \sqrt{n}} \lambda_{n}(x, t) d_{t}\left(-\bigvee_{t}^{x}\left(g_{x}\right)\right) d t \\
\leq & \bigvee_{x-x / \sqrt{n}}^{x}\left(g_{x}\right) \frac{(\alpha-\beta x)^{2}+n x(1-x)}{(n+\beta)^{2}\left(\frac{x}{\sqrt{n}}\right)^{2}} \\
& +\frac{(\alpha-\beta x)^{2}+n x(1-x)}{(n+\beta)^{2}} \int_{0}^{x-x / \sqrt{n}} \frac{1}{(x-t)^{2}} d_{t}\left(-\bigvee_{t}^{x}\left(g_{x}\right)\right) d t .
\end{aligned}
$$


134

RÜYA ÜSTER AND ERTAN İBIKKLI

Furthermore, again by partial integration,

$$
\begin{aligned}
\int_{0}^{x-x / \sqrt{n} \frac{1}{(x-t)^{2}} d_{t}\left(-\bigvee_{t}^{x}\left(g_{x}\right)\right) d t=} & -\frac{1}{\left(\frac{x}{\sqrt{n}}\right)^{2}} \bigvee_{x-x / \sqrt{n}}^{x}\left(g_{x}\right)+\frac{1}{x^{2}} \bigvee_{0}^{x}\left(g_{x}\right) \\
& +\int_{0}^{x-x / \sqrt{n}} \frac{2}{(x-t)^{3}} \bigvee_{t}^{x}\left(g_{x}\right) d t
\end{aligned}
$$

Putting $t=x-\frac{x}{\sqrt{u}}$ in the last integral, we obtain

$$
\int_{0}^{x-x / \sqrt{n}} \frac{2}{(x-t)^{3}} \bigvee_{t}^{x}\left(g_{x}\right) d t=\frac{1}{x^{2}} \int_{1}^{n} \bigvee_{x-x / \sqrt{u}}^{x}\left(g_{x}\right) d u=\frac{1}{x^{2}} \sum_{k=1}^{n} \bigvee_{x-x / \sqrt{k}}^{x}\left(g_{x}\right)
$$

Consequently,

$$
\begin{aligned}
\left|I_{1}(n, x)\right| \leq & \bigvee_{x-x / \sqrt{n}}^{x}\left(g_{x}\right) \frac{(\alpha-\beta x)^{2}+n x(1-x)}{(n+\beta)^{2}\left(\frac{x}{\sqrt{n}}\right)^{2}} \\
& +\frac{(\alpha-\beta x)^{2}+n x(1-x)}{(n+\beta)^{2}} \\
& \times\left\{-\frac{1}{\left(\frac{x}{\sqrt{n}}\right)^{2}} \bigvee_{x-x / \sqrt{n}}^{x}\left(g_{x}\right)+\frac{1}{x^{2}} \bigvee_{0}^{x}\left(g_{x}\right)\right. \\
& \left.+\frac{1}{x^{2}} \sum_{k=1}^{n} \bigvee_{x-x / \sqrt{k}}^{x}\left(g_{x}\right)\right\} \\
\leq & \frac{(\alpha-\beta x)^{2}+n x(1-x)}{(n+\beta)^{2} x^{2}}\left\{\bigvee_{0}^{x}\left(g_{x}\right)+\sum_{k=1}^{n} \bigvee_{x-x / \sqrt{k}}^{x}\left(g_{x}\right)\right\}
\end{aligned}
$$

Using a similar method as for estimating $\left|I_{3}(n, x)\right|$, we get

$$
\left|I_{3}(n, x)\right| \leq \frac{(\alpha-\beta x)^{2}+n x(1-x)}{(n+\beta)^{2}(1-x)^{2}}\left\{\bigvee_{x}^{1}\left(g_{x}\right)+\sum_{k=1}^{n} \bigvee_{x}^{x+(1-x) / \sqrt{n}}\left(g_{x}\right)\right\}
$$


Hence from (3.4)-(3.6), it follows that

$$
\begin{aligned}
\left|B_{n}^{(\alpha, \beta)}\left(g_{x}, x\right)\right| \leq & \left|I_{1}(n, x)\right|+\left|I_{2}(n, x)\right|+\left|I_{3}(n, x)\right| \\
\leq & \frac{1}{n-1} \sum_{k=2}^{n} \bigvee_{x-x / \sqrt{k}}^{x+(1-x) / \sqrt{k}}\left(g_{x}\right) \\
& +\frac{(\alpha-\beta x)^{2}+n x(1-x)}{(n+\beta)^{2} x^{2}}\left\{\bigvee_{0}^{x}\left(g_{x}\right)+\sum_{k=1}^{n} \bigvee_{x-x / \sqrt{k}}^{x}\left(g_{x}\right)\right\} \\
& +\frac{(\alpha-\beta x)^{2}+n x(1-x)}{(n+\beta)^{2}(1-x)^{2}} \\
& \times\left\{\bigvee_{x}^{1}\left(g_{x}\right)+\sum_{k=1}^{n} \bigvee_{x-x / \sqrt{k}}^{x+(1-x) / \sqrt{k}}\left(g_{x}\right)\right\}
\end{aligned}
$$

Because $\frac{1}{x^{2}}+\frac{1}{(1-x)^{2}} \leq \frac{1}{x^{2}(1-x)^{2}}, x \in[0,1]$

$$
\begin{aligned}
\left|B_{n}^{(\alpha, \beta)}\left(g_{x}, x\right)\right| \leq & \frac{(\alpha-\beta x)^{2}+n x(1-x)}{(n+\beta)^{2}(1-x)^{2} x^{2}}\left\{\bigvee_{0}^{1}\left(g_{x}\right)+\sum_{k=1}^{n} \bigvee_{x-x / \sqrt{k}}^{x+(1-x) / \sqrt{k}}\left(g_{x}\right)\right\} \\
& +\frac{1}{n-1} \sum_{k=2}^{n} \bigvee_{\substack{x-x / \sqrt{k} \\
\text { n) }}}^{x+(1-x) / \sqrt{k}}\left(g_{x}\right) .
\end{aligned}
$$

On the other hand, note that $\bigvee_{0}^{1}\left(g_{x}\right) \leq \sum_{k=1}^{n} \underset{\substack{x-x / \sqrt{k} \\ \bigvee}}{\operatorname{xa}(1-x) / \sqrt{k}}\left(g_{x}\right)$, so that

$$
\begin{aligned}
\left|B_{n}^{(\alpha, \beta)}\left(g_{x}, x\right)\right| \leq & 2\left[\frac{(\alpha-\beta x)^{2}+n x(1-x)}{(n+\beta)^{2}(1-x)^{2} x^{2}}\right]\left\{\sum_{k=1}^{n} \bigvee_{x-x / \sqrt{k}}^{x+(1-x) / \sqrt{k}}\left(g_{x}\right)\right\} \\
& +\frac{1}{n-1} \sum_{k=2}^{n} \bigvee_{x-x / \sqrt{k}}^{x+(1-x) / \sqrt{k}}\left(g_{x}\right) .
\end{aligned}
$$

Noting that $\frac{1}{n-1} \leq \frac{(\alpha-\beta x)^{2}+n x(1-x)}{(n+\beta)^{2}(1-x)^{2} x^{2}}$, for $n>1$, we have

$$
\left|B_{n}^{(\alpha, \beta)}\left(g_{x}, x\right)\right| \leq 3 \frac{(\alpha-\beta x)^{2}+n x(1-x)}{(n+\beta)^{2}(1-x)^{2} x^{2}}\left\{\sum_{k=1}^{n} \bigvee_{x-x / \sqrt{k}}^{x+(1-x) / \sqrt{k}}\left(g_{x}\right)\right\}
$$

Putting (2.1) and (3.6) in (3.3), we deduce the required result (1.1). Thus the proof of our theorem is finally complete.

\section{REFERENCES}

[1] Bojanic, R. and Vuilleumier, M. On the rate of convergence of Fourier-Legendre series of functions of bounded variation, 1981.

[2] Cheng, F. On the rate of convergence of Bernstein polynomials of functions of bounded variation, 1983. 
[3] Zeng. X. M. and Chen, W. On the rate of convergence of the generalized Durrmeyer type operators for functions of bounded variation. J. Approx. Theory 102:1-12, 2000.

[4] Guo, S. S. On the rate of convergence of Durrmeyer operator for functions of bounded variation. Journal of Approximation Theory 51, 183-197, 1987.

[5] Bernstein, S. N. Demonstration du Théoreme de Weierstrass fondéee sur le calcul des probabilités. Comm. Soc. Math. 13:1-2, 1912.

[6] Stancu, D.D. Approximation of functions by means of a new generalized Bernstein operator, Calcolo 20 211-229, 1983

[7] Shiryayev, A.N. Probability. Springer-Verlag, New York, 1984.

[8] Karsli, H. and Ibikli, E. Rate of Convergence of Chlodowsky-Type Bernstein Operators for Functions of Bounded Variation, Numerical Functional Analysis and Optimization, 28:3-4, $367-378,2007$

[9] Zeng X.-M., Bounds for Bernstein basis functions and Meyer-König-Zeller basis functions, J. Math. Anal. Appl. 219:364-376, 1998.

İsTANBUL-TURKEY

E-mail address: ruya.uster@istanbul.edu.tr

ANKARA-TURKEY

E-mail address: ibikli@ankara.edu.tr 